\title{
Statistical and subjective interpretations of probability in quantum-like models of cognition and decision making
}

\author{
Emmanuel Haven ${ }^{\mathrm{a}}$, Andrei Khrennikov ${ }^{\mathrm{b}}$ \\ ${ }^{a}$ School of Management and IQSCS, University of Leicester, UK \\ ${ }^{b}$ International Center for Mathematical Modeling in Physics and Cognitive Sciences \\ Linnaeus University, Växjö, Sweden
}

\begin{abstract}
The paper starts with an introduction to the basic mathematical model of classical probability (CP), i.e. the Kolmogorov (1933) measure-theoretic model. Its two basic interpretations are discussed: statistical and subjective. We then present the probabilistic structure of quantum mechanics (QM) and discuss the problem of interpretation of a quantum state and the corresponding probability given by Born's rule. Applications of quantum probability (QP) to modeling of cognition and decision making (DM) suffer from the same interpretational problems as QM. Here the situation is even more complicated than in physics. We analyze advantages and disadvantages of the use of subjective and statistical interpretations of QP. The subjective approach to QP was formalized in the framework of Quantum Bayesianism (QBism) as the result of efforts from C. Fuchs and his collaborators. The statistical approach to QP was presented in a variety of interpretations of QM, both in nonrealistic interpretations, e.g., the Copenhagen interpretation (with the latest version due to A. Plotnitsky), and in realistic interpretations (e.g., the recent Växjö interpretation). At present, we cannot make a definite choice in favor of any of the interpretations. Thus, quantum-like DM confronts the same interpretational problem as quantum physics does.
\end{abstract}

Key words: Classical and quantum probability, subjective and statistical probabilities, interpretation, decision making, probability update, QBism, Växjö interpretation. 


\section{Introduction}

Recently the mathematical formalism of quantum mechanics ('QM' henceforth), especially the apparatus of quantum probability ('QP' henceforth), started to be widely used outside of physics for the modeling of cognition and decision making ('DM' henceforth) in psychology, psychophysics, economics, finance, political science and the wider social sciences, see the basic monographs (Ezhov \& Berman (2003); Khrennikov (2010); Busemeyer \& Bruza (2012); Bagarello (2012); Haven \& Khrennikov (2013); Asano et al. (2015)) and the recent review articles (Busemeyer et al. (2014); Plotnitsky (2014)) and references therein; as well as a selection of some recent publications relevant to probabilistic foundations (de Barros \& Suppes (2009); de Barros \& Oas (2014); Atmanspacher \& Filk (2014b)). Such models can be called quantum-like to distinguish them from genuine quantum physical models. In quantum-like models we explicitly do not refer to quantum physical processes which (may) take place in biological systems, in particular, in the brains of decision makers. Our modeling is based on the quantum-like paradigm (see Khrennikov (2010)): the process of DM within bio-systems with a complex information structure (e.g., by humans) is described by QP. This paradigm has an empirical origin: there is plenty of probabilistic data available, e.g., in cognitive psychology and psychophysics which exhibits the violation of the basic laws of classical probability (' $\mathrm{CP}$ ' henceforth), e.g. the formula of total probability ('FTP' henceforth) (see, e.g., Khrennikov (2010); Busemeyer \& Bruza (2012)) or the Bell inequality (see, e.g., Khrennikov (2010); Conte et al. (2008)). Violations of the laws of classical probability theory by quantum physical systems were discussed by many authors (see, e.g., Feynman \& Hibbs (1965), or Khrennikov (2009)). This situation is well modeled by QP based on Born's rule connecting complex probability amplitudes (complex state vectors, wave functions) with real probabilities. One could make the argument that it could be useful to try to model similar violations of classicality outside of physics with the aid of the same calculus. However, this apparent similarity does surely not guarantee that the formalism which worked so well in one domain of science, in physics, will work as well in other domains. Its fruitfulness can be justified by successful applications. We remark that the situation does not differ so much from physics. QM is held in very high esteem because it works so well. On the other hand, the project on the justification of the impossibility of its reduction to classical statistical

models (see, e.g., von Neumann (1955), or Bell (1987)), still has not been 
completed (Khrennikov (2008); Khrennikov (2010)) ${ }^{1}$.

Applications of the quantum formalism and, in particular, QP to model cognition and DM can be characterized as really successful (see Ezhov \& Berman (2003); Khrennikov (2010); Busemeyer \& Bruza (2012); Bagarello (2012); Haven \& Khrennikov (2013); Asano et al. (2015); Busemeyer et al. (2014); Plotnitsky (2014)) for various studies. At the same time one has to be cautious. One can not expect that the whole body of QM would be useful for such applications. Moreover, it may happen that some cognitive or social phenomena would not be covered completely by the standard quantum formalism (cf. Khrennikov et al. (2014a)). It may well be that more general probabilistic models have to be developed (see Khrennikov (2010)).

We remark that although QM works very well, its theoretical and philosophic justification is far from complete. In particular, QM suffers from the problem of interpreting a quantum state (wave function) (see, for example, Plotnitsky (2006); Plotnitsky (2009); Khrennikov (2009)). The present situation is characterized by a huge diversity of interpretations and this cannot be considered as acceptable. Since QM is about probabilities (it does not predict the individual outputs of measurements), the problem of the interpretation of a quantum state is very closely related to the problem of the interpretation of a probability. In this paper we analyze the probability interpretation dimension of QM in connection to DM and to applications of QM's cognitive psychology. Of course, the state interpretation problem is not reduced to the interpretation of probability given by Born's rule. Thus, in this paper we shall treat the problem of an interpretation of QM only partially.

In any scientific theory one has to distinguish the formalism and its interpretation. The mathematical formalism of modern classical probability theory is based on measure theory (see Kolmogorov (1933)). However, it is interesting (and it is maybe not so well known) that Kolmogorov not only developed the commonly used mathematical formalism of probability theory (including purely mathematical contributions such as Kolmogorov's theorem on the existence of the probability measure for a stochastic process and the

\footnotetext{
${ }^{1}$ The von Neumann theorem was strongly criticized for its un-physical assumptions, by Margenau, Bell and Ballentine. Experimental verification of a violation of Bell's inequality is a very challenging project, since it is very difficult to perform the loophole free experiment producing statistically acceptable data (see, e.g., Khrennikov et al. (2014b)) for analysis and Hensen et al. (2015) for the most recent success in this area.
} 
strong law of large numbers), but he also endowed his theory with a special interpretation of probability, i.e. the Kolmogorov interpretation. Thus, just as in any theory, in Kolmogorov's theory one has to distinguish between the mathematical formalism and its interpretation. Besides the genuine Kolmogorov interpretation, his formalism can be interpreted in different ways. Among the huge variety of interpretations of probability, we point to two of the most known and applicable interpretations:

- ST statistical interpretation (Kolmogorov (1933); von Mises (1957); Feller (1968); Khrennikov (2009); Rocchi (2014); Plotnitsky (2009));

- SUB subjective (Ramsey (1931); de Finetti (1990); Savage (1954); Bernardo \& Smith (1994); Rocchi (2003); Rocchi (2014)).

ST: probability is a characteristic of a "mass phenomenon, or a repetitive event, or simply a long sequence of observations (see von Mises (1957)). Here probability cannot be assigned to an individual event. The condition of the event's repeatability (in theory infinite repeatability) is crucial. Numerically, probability is defined as the limit of frequencies (in von Mises' theory this is the definition of probability and in Kolmogorov's theory it is a consequence of the law of large numbers).

SUB: probability is assigned to an individual event $A$ and it represents the degree of the personal belief in the non/occurrence of $A$. Thus, such probability is private and individual.

Now we want to couple the interpretations of a quantum state and the corresponding probability given by Born's rule. This coupling leads to two important interpretations of a quantum state:

- STQ statistical (ensemble) interpretation (Bohr, Pauli, Dirac, von Neumann, Einstein, Schrödinger, de Broglie, Bohm, Margenau, Ballentine $)^{2}$ (see, e.g., Plotnitsky (2006); Plotnitsky (2009); Khrennikov (2009));

- QBism quantum Bayesian (subjective) interpretation (see, e.g., Fuchs (2011); Fuchs \& Schack (2013); Fuchs \& Schack (2015)).

\footnotetext{
${ }^{2}$ It is interesting that very different interpretations of QM can keep the same interpretation of probability. For example, both the Copenhagen interpretation and the de Broglie-Bohm interpretation treat probability statistically
} 
STQ can be characterized by a diversity of 'sub-interpretations' depending on whether the results of observations can be treated independently of the measurement procedures or not (the problem of realism in QM). QBism was created recently and it has yet just one version. As we can see from the STQlist, this interpretation dominates in the quantum community. In terms of recent contributions to its development we can mention the Växjö interpretation (see Khrennikov (2002)); the realist contextual statistical interpretation; the statistical Copenhagen interpretation invented by A. Plotnitsky ${ }^{3}$ and the non-realist statistical interpretation. At the same time, the recent quantum information revolution stimulated the dissemination of QBism. However, it is still considered as an exotic 'non-physical' interpretation of $\mathrm{QM}^{4}$.

Now, suppose one applies QP to model the DM-process, e.g., in psychology, psychophysics or economics. She/he is immediately confronted with the cognitive/mental version of the problem of the interpretation of quantum states and probabilities: the problem which was not solved in quantum physics and was 'imported' from it to cognitive science, DM, psychology or psychophysics. Moreover, novel applications induce novel interpretational issues. Our aim is to analyze the specifics of the use of STQ and QBism to model cognition and DM. The problem is very complex and at the moment we are only able to present some reasons in favor of and against each of these interpretations. We hope that our analysis will stimulate the further emergence of foundational studies on the problem of the interpretations of mental states (belief states) and the corresponding probabilities in QP-modeling of DM and problem solving.

\section{Classical probability}

In the 19th century George Boole wrote the book "An Investigation of the Laws of Thought on Which are Founded the Mathematical Theories of Logic and Probabilities" (Boole (1958)). This was the first mathematical model of the process of thinking based on the laws of reasoning which nowadays are known as Boolean logic. This logic, also known as classical logic, plays

\footnotetext{
${ }^{3}$ It was presented in his talk at the conference "Quantum Theory: from Foundations to Technologies", Växjö -2015.

${ }^{4} \mathrm{QBism}$ is often labeled as one of the neo-Copenhagen interpretations of QM. This is a totally wrong viewpoint on QBism (see, for example, Mermin (2014)).
} 
a crucial role in information theory, DM, computer science and artificial intelligence and digital electronics.

Boolean logic serves as the basis of modern probability theory (Kolmogorov (1933)), which is based on the representation of events by sets, subsets of some set $\Omega$, the sample space, or space of elementary events. The system of sets representing events, say $\mathcal{F}$, matches with the operations of Boolean logics; $\mathcal{F}$ is a so-called $\sigma$-algebra of $\operatorname{sets}^{5}$. It is closed with respect to the (Boolean) operations of (countable) union, intersection, and complement (or in logical terms 'and, or, not'). Thus, by applying any theorem of probability theory, e.g., the central limit theorem, we use classical Boolean logic.

\section{Mathematical formalism.}

The set-theoretic model of probability was presented by Kolmogorov (1933). It is based on the following two natural (from the viewpoint of classical logic) axioms:

- AK1) events are represented as elements of a $\sigma$-algebra and operations for events are described by Boolean logic;

- AK2) probability is represented as a probability measure.

For the convenience of the reader, we present the definition of a probability measure: $p$ is a (countably) additive function on a $\sigma$-algebra $\mathcal{F}$ : $p\left(\cup_{j=}^{\infty} A_{j}\right)=\sum_{j=}^{\infty} p\left(A_{j}\right)$, for $A_{j} \in \mathcal{F}, A_{i} \cap A_{j}=\emptyset, i \neq j$, which is valued in $[0,1]$ and normalized by 1 . A triple $\mathcal{P}=(\Omega, \mathcal{F}, p)$ is called the (Kolmogorov) probability space.

We also remind the definition of a random variable as a measurable function, $a: \Omega \rightarrow \mathbb{R}$. In applications of $\mathrm{CP}$, e.g. to classical statistical physics and thermodynamics or to cognitive modeling, psychology and psychophysics, random variables represent observables.

We point out that modern probability theory is an axiomatic theory, in the same way, as say geometry is. The history of the development of geometry showed us that one can play with axiomatic systems and develop a variety of geometric models.

\section{Conditional probability and formula of total probability.}

\footnotetext{
${ }^{5}$ Here the symbol $\sigma$ encodes "countable". In American terminology such systems of subsets are called $\sigma$-fields.
} 
One of the basic laws of the Kolmogorovian model, the formula of total probability ('FTP' henceforth), will play a very important role in our further considerations. Before we go to the FTP, we point to the exceptional role which is played by conditional probability in the Kolmogorovian model. This sort of probability is not derived in any way from the 'usual probability'. Conditional probability is per definition given by the Bayes formula:

$$
P(B \mid C)=P(B \cap C) / P(C), P(C)>0 .
$$

By Kolmogorov's interpretation it is the probability of an event $B$ to occur under the condition that an event $C$ has occurred. One can immediately see that this formula is one of the strongest exhibitions of the Boolean structure of the model: one cannot even assign conditional probability to an event without using the Boolean operation of intersection. Let us consider a countable family of disjoint sets $H_{k}$ belonging to $\mathcal{F}$ such that their union is equal to $\Omega$ and $P\left(H_{k}\right)>0, k=1, \ldots$. Such a family is called a partition of the space $\Omega$.

Theorem 1 Let $\left\{H_{k}\right\}$ be a partition. Then, for every set $B \in \mathcal{F}$, the following formula of total probability holds

$$
p(B)=\sum_{k} p\left(H_{k}\right) p\left(B \mid H_{k}\right)
$$

This formula plays a crucial role in classical decision theory based on the Bayesian procedure for probability updating (PU). The events $H_{k}$ are treated as hypotheses and the probabilities $p\left(H_{k}\right)$ as prior probabilities. Especially interesting for us is the case, where a partition is induced by a discrete random variable $a$ taking values $\left\{\alpha_{k}\right\}$. Here, $H_{k}=\left\{\omega \in \Omega: a(\omega)=\alpha_{k}\right\}$. Let $b$ be another discrete random variable. It takes values $\left\{\beta_{j}\right\}$. For any $\beta_{j}$, we have $p\left(b=\beta_{j}\right)=\sum_{k} p\left(a=\alpha_{k}\right) P\left(b=\beta_{j} \mid a=\alpha_{k}\right)$. Here $p\left(a=\alpha_{k}\right)=p\left(H_{k}\right)$.

\section{Bell's inequality in Wigner's form.}

We start with a trivial application of classical probability theory exploring the additivity of probability and its non-negativity.

Consider three events $A, B, C$. It is convenient to use the notations

$$
A \equiv A_{+}, \complement \subset \equiv A_{-}, B \equiv B_{+}, \complement\left[B \equiv B_{-}, C \equiv C_{+}, \complement C C \equiv C_{-},\right.
$$

where, for a set $O$, the symbol $\complement O$ denotes its complement, i.e., $\complement O=\Omega \backslash O=$ $\{\omega \in \Omega: \omega \notin O\}$. 
Theorem 2. (Bell-Wigner inequality) The following inequality holds:

$$
P\left(A_{+} \cap B_{+}\right)+P\left(B_{-} \cap C_{+}\right) \geq P\left(A_{+} \cap C_{+}\right) .
$$

Proof. For each term of (4), we shall use the equality $P(A)=P(A \cap$ $B)+P(A \cap \complement B)$. For the first term, the event $A_{+} \cap B_{+}$plays the role of $A$ in this equality and the event $C_{+}$plays the role of $B$. We have

$$
P\left(A_{+} \cap B_{+}\right)=P\left(A_{+} \cap B_{+} \cap C_{+}\right)+P\left(A_{+} \cap B_{+} \cap C_{-}\right) .
$$

In the same way we obtain

$$
\begin{aligned}
& P\left(B_{-} \cap C_{+}\right)=P\left(B_{-} \cap C_{+} \cap A_{+}\right)+P\left(B_{-} \cap C_{+} \cap A_{-}\right), \\
& P\left(A_{+} \cap C_{+}\right)=P\left(A_{+} \cap C_{+} \cap B_{+}\right)+P\left(A_{+} \cap C_{+} \cap B_{-}\right) .
\end{aligned}
$$

By adding the first two equalities we come to the expression

$$
P\left(A_{+} \cap B_{+} \cap C_{+}\right)+P\left(A_{+} \cap B_{+} \cap C_{-}\right)+P\left(B_{-} \cap C_{+} \cap A_{+}\right)+P\left(B_{-} \cap C_{+} \cap A_{-}\right) .
$$

Commutativity of the operation of intersection implies that $P\left(A_{+} \cap B_{+}\right)+$ $P\left(B_{-} \cap C_{+}\right)$equals to $P\left(A_{+} \cap C_{+}\right)$plus a non-negative term. Hence, (4) holds.

This inequality plays a fundamental role in modern quantum physics (in spite of its mathematical triviality). It is one of the so called 'Bell type' inequalities. In QM it is derived from two basic physical assumptions: locality and realism.

In the above considerations the assumption of realism $\mathrm{R}$ was encoded in the possibility to represent events by subsets of some set $\Omega$. This setrepresentation provides objectivization of events; the events $A, B, C$ exist independently of measurements performed by an observer.

The assumption of locality $\mathrm{L}$ was encoded in the possibility to operate with only three events, $A, B, C$ (and their complements). In general one has to start with indexes $a, b, c$ determining experimental settings, orientations of beam splitters. In the local model, the events are completely determined by corresponding settings, $A \equiv A_{a}, B \equiv B_{b}, C \equiv C_{c}$. In the nonlocal model, in the experiment with the pair of orientations $(x, y)$ the events depend on this pair of orientations, e.g., $A_{(a, y)}$, where $y=b, c$. In fact, in the Bell scheme the physical space time is not present at all. Therefore, it is more natural 
to speak about (non)contextuality (see Khrennikov (2010); Dzhafarov \& Kujala (2013, 2014 a, b)).

Violation of 'Bell type' inequalities by QP is the main reason to reject the so-called local realism and it led to the cardinal reconsideration of the foundation of physics. One has to accept that either nature cannot be described by a realist model or it is nonlocal: NR or NL. We remark that here 'or' is non-exclusive. A part of the quantum community really believes that nature combines NR and NL. However, the majority selected either NR and L, e.g., Zeilinger (2010), or R and NL, e.g., Gisin \& Aspect (2014).

To derive Bell's inequality (under the assumption of locality) as a consequence of the use of the classical probability model (based on Kolmogorov axiomatics), all events can be represented in the set-theoretic framework and it is possible to set a single measure to define probabilities of all those events and, hence, by the laws of Boolean algebra of their intersections. Thus, from this viewpoint the violation of the 'Bell type' inequalities is just a sign of the impossibility to use Boolean algebra for some events and/or the impossibility to define the probability measure serving for all such events. By exploring this interpretation (NR and L) of the violation of 'Bell type' inequalities in cognitive science and psychology one says that cognitive systems and, in particular, humans use non-Boolean logic in the process of DM leading to nonclassical probabilistic statistics. Another possibility is nonlocality (more general contextuality) of brain's functioning. Such a brain can proceed by using classical Boolean logic and data processed by it can be represented in Kolmogorov's model. We remark that in this cognitive framework nonlocality is not so mystical as in quantum physics. The brain is the very small physical object comparing with the velocity of propagation of electromagnetic waves. Its different areas, can actively communicate and create nonlocal probabilistic settings. There is no need in action at a distance (cf. de Barros \& Suppes (2009)).

\section{Statistical and subjective interpretations of probability.}

Kolmogorov's probability theory (as any scientific theory) consists of two parts: the mathematical formalism and interpretation. Now we turn to its interpretation:

"[. . . ] we may assume that to an event $A$, which may or may not occur under conditions $\Sigma$, is assigned a real number $P(A)$ which has the following characteristics:

- (a) one can be practically certain that if the complex of conditions 
$\Sigma$ is repeated, a large number of times, $N$, then if $n$ be the number of occurrences of event $A$, the ratio $n / N$ will differ very slightly from $P(A)$;

- (b) if $P(A)$ is very small, one can be practically certain that when conditions $\Sigma$ are realized only once, the event $A$ would not occur at all."

The (a)-part of this interpretation is nothing else than the frequency interpretation of probability (see von Mises (1957)). This is the essence of the 'statistical interpretation of probability' which is mathematically justified by the law of large numbers (a theorem in the Kolmogorov measure-theoretic mathematical model).

The (b)-part is a more complicated statement. The referring to "to be practically certain" that "the event $A$ would not occur at all" can be treated as a subjective element of Kolmogorov's interpretation of probability (see also the discussion below on Cournot's principle and Bernoulli's moral certainty). Since "practically" depends on the viewpoint of a decision maker, this is a step, although small (since here probability is treated objectively with objectification through calculation of frequencies), towards the subjective interpretation of probability by de Finetti (1990). For Kolmogorov, the objectivity of statistical probability is encoded in the complex of conditions (context) $\Sigma$, it is its objective property, determined by its repeatability.

\section{Cournot's principle.}

The (b)-part of Kolmogorov's interpretation of probability is also known as Cournot's principle. Its first version is due to J. Bernoulli (1713) who related mathematical probability to moral certainty/impossibility: "Something is morally certain if its probability is so close to certainty that the shortfall is imperceptible." "Something is morally impossible if its probability is no more than the amount by which moral certainty falls short of complete certainty." In spite of our above remark that there is a subjective element in the (b)- part of Kolmogorov's interpretation (and Cournot's principle) , setting the level of moral impossibility, those who used this principle treated probability objectively. Subjectivists, as de Finetti, rejected it.

\section{Contextuality of Kolmogorov theory.}

This reference to $\Sigma$ is very important for our further considerations. Kolmogorov pointed out that each probability space is determined by its own 
complex of conditions (context) $\Sigma$. For example, he definitely would not be surprised by the violation of the Bell-type inequalities if the pairs of events occur under different contexts, e.g. the pair of events $\left(A_{+}, B_{+}\right),\left(B_{-}, C_{+}\right),\left(A_{+}, C_{+}\right)$ in the Bell-Wigner inequality occur for three different contexts $\Sigma_{j}, j=1,2,3$. In general, each of these contexts determines its own probability space $\mathcal{P}_{\Sigma_{j}}=$ $\left(\Omega_{\Sigma_{j}}, \mathcal{F}_{\Sigma_{j}}, p_{\Sigma_{j}}\right)$. Since the Bell-Wigner inequality was proven by working in a single Kolmogorov probability framework, the possibility of its violation in a multi-space framework is not surprising.

\section{Classical decision making through the Bayesian probability up- date}

The classical scheme of DM is based on the Bayesian probability update ('PU' henceforth). There is a set of states of nature $\Theta=\left\{\theta_{1}, \ldots, \theta_{m}\right\}$ (or 'states of mind' in applications to cognition and psychology); a random variable $A$ is given and it is taking values from the set $X=\left\{x_{1}, \ldots, x_{m}\right\}$; and for each state of nature $\theta$ one can get the probability distribution $\pi(x \mid \theta), x \in X$.

As a starting point, say Alice assigns the probabilities to possible states of nature, $\pi(\theta)$. It can be considered as the probability distribution of a random variable $B$.

Alice then measures the random variable and updates the prior probability distribution on the basis of information gained from this concrete result of measurement. The classical PU is based on the Bayes rule:

$$
\pi(\theta \mid x)=\frac{\pi(x \mid \theta) \pi(\theta)}{p(x)}, p(x)=\sum_{\theta} \pi(\theta) \pi(x \mid \theta),
$$

where the last equality is FTP (2).

In the formalism of PU and DM, instead of a collection $\Theta$ of states of nature (mind), we can consider a collection of hypotheses $\left(H_{k}\right)$ forming the disjoint partition of the sample space $\Omega$. The Bayesian PU can be used to update the probabilities of these hypotheses as the evidence $A=x$ appears: $\pi\left(H_{k} \mid x\right)=\frac{\pi\left(x \mid H_{k}\right) \pi\left(H_{k}\right)}{p(x)}, p(x)=\sum_{k} \pi\left(H_{k}\right) \pi\left(x \mid H_{k}\right)$.

Subjective and frequentist interpretations of classical Bayesian inference.

Bayesian inference is simple mathematically. However, its interpretation reflects the diversity of interpretations of probability. Originally (by Bayes) all probabilities in (9) were interpreted as subjective probabilities (see de 
Finetti (1990)). The prior probabilities $\pi(\theta)$ represent Alice's degrees of belief that the real state of nature (mind) is $\theta$, prior to the information about the value $x$ of $A$. The same is valid for the conditional probabilities $\pi(x \mid \theta)$, likelihoods. These are degrees of Alice's belief that $A$ would take the value $x$ if the state $\theta$ were realized. The output $\pi(\theta \mid x)$ of PU (9) is the degree of belief that the state of nature is $\theta$, in light of the information that $A=x$. This subjective probability viewpoint on the Bayesian PU is widely used and is known as Bayesianism.

However, the mathematical formula (9) can be interpreted in a totally different way, in the frequentist framework (see von Mises (1957), or Rocchi (2003); Rocchi (2014)). Here probabilities are assigned not to individual events, but they rather represent frequencies of realization of parameters in a long series of experiments. The values of the prior probabilities $\pi(\theta)$ as well as the likelihoods $\pi(x \mid \theta)$ are estimated on the basis of statistical data available before the measurements of $A$. The output of $(9), \pi(\theta \mid x)$, gives the probability that the state of nature is $\theta$, conditioned on the result $A=x$, i.e. in a long sequence of experiments, the frequency of realization of $\theta$ conditioned on the value $x$ of $A$ approaches $\pi(\theta \mid x)$ (see von Mises (1957)).

For a subjective Bayesian, the probability distribution given by (9) reflects knowledge about the present state of nature (mind) after collecting data. For a frequentist, (9) does not reflect such knowledge: it is not about the 'present state', as this state is the only unknown parameter, and $\pi(\theta \mid x)$ is its probability distribution.

The subjective approach can be used in DM to make an individual decision. Suppose that the parameter $\theta$ is dichotomous, $\theta=\theta_{1}, \theta_{2}$. The odds in favor of an event is given by the ratio of the probability that it will occur to the probability that it will not; so one sets

$$
O\left(\theta_{1}\right)=\frac{\pi\left(\theta_{1} \mid x\right)}{\pi\left(\theta_{2} \mid x\right)}=\frac{\pi\left(x \mid \theta_{1}\right) \pi\left(\theta_{1}\right)}{\pi\left(x \mid \theta_{2}\right) \pi\left(\theta_{2}\right)} .
$$

If

$$
O\left(\theta_{1}\right)>1
$$

then Alice decides that the state $\theta=\theta_{1}$. In the opposite case she takes $\theta=\theta_{2}$.

The frequentist approach can be used in DM as well, but for decisions which can be repeated for many trials (in theory for infinitely many trials). Here the odds-function (10) is used not for the individual DM, but for estimating the frequency of realization of the state of the world (mind) $\theta_{1}$ 
compared to the state of the world (mind) $\theta_{2}$. Of course, not every DM can be embedded into the frequentist framework.

\section{Cromwell rule.}

We point to one important feature of the classical PU and, hence, the classical DM model. For some state of nature (including the mind): if a decision maker, Alice, assigned to some state $\theta$ the prior probability zero, then any PU would lead again to a zero posterior probability. In this case the Bayesian PU simply idles. It can thus be argued that such PU excludes any possibility to come to novel creative decisions. In the same way if $\pi(\theta)=1$, then $p(x)=\pi(x \mid \theta)$ and $\pi(\theta \mid x)=1$. Here again, the Bayesian PU simply idles. To escape Bayesian idling, one has to follow the so-called Cromwell rule (see Lindley (1991)): "the use of prior probabilities of 0 or 1 should be avoided, except when applied to statements that are logically true or false." Thus, all possibilities (even 'practically impossible') have to be taken into account by assigning them, although very small but nevertheless, nonzero probabilities $\epsilon>0$. However, the use of the Cromwell rule leads to huge lists of possible states of nature (mind) which all have to be taken into account in the process of PU. It generates incredible computational difficulties. In the situation when a decision has to be made as quickly as possible, the applicability of the Bayesian PU is really questionable. Therefore, it would be attractive to proceed without this rule and at the same time to escape Bayesian idling. Such a possibility is provided by the quantum scheme of DM which is based on a generalization of the Bayesian PU, see the next section.

Thus, a big enough state space is an important first step in the Bayesian approach to DM. From the start, we have to account even for the most inconceivable possibilities by considering the corresponding states of nature (e.g., that the moon is made of green cheese (see Lindley (1991))). In the quantum model, though, we are free to assign to them zero priors. Of course, the invariance of the extreme probabilities, zero and one, with respect to the Bayesian PU is just one of the symptoms of classical Boolean logic in the process of DM when modeled with the aid of CP. 


\section{Quantum states, observables, and probabilities}

The state space of a quantum system is based on a complex Hilbert space ${ }^{6}$. Denote it by $H$. This is a complex linear space endowed with a scalar product, a positive-definite non-degenerate Hermitian form. Denote the latter by $\langle\cdot \mid \cdot\rangle$. It determines the norm on $H,\|\phi\|=\sqrt{\langle\phi \mid \phi\rangle}$.

A reader who does not feel comfortable with the abstract framework of functional analysis can simply proceed with the Hilbert space $H=\mathbb{C}^{n}$, where $\mathbb{C}$ is the set of complex numbers, and the scalar product $\langle u \mid v\rangle=$ $\sum_{i} u_{i} \bar{v}_{i}, u=\left(u_{1}, \ldots, u_{n}\right), v=\left(v_{1}, \ldots, v_{n}\right)$. Instead of linear operators, one can consider matrices.

Pure quantum states are represented by normalized vectors, $\psi \in H$ : $\|\psi\|=1$.

In the standard QM (Dirac-von Neumann formalism) a quantum observable $A$ is represented by a Hermitian operator $\hat{A}$. Let there be given a state $\psi$ and a quantum observable with the spectral decomposition $\hat{A}=\sum_{i} a_{i} P_{i}$, where $a_{i}$ are eigenvalues of $\hat{A}$, and $P_{i}$ are orthogonal projectors onto the corresponding eigen-subspaces. The system of mutually orthogonal projectors $\left(P_{i}\right)$ is known as the orthogonal partition of the unit operator: $\sum_{i} P_{i}=$ $I, P_{i} \perp P_{k}, i \neq k$.

We remark that by the spectral postulate of QM, in any measurement of $A$, one can get only one of its eigenvalues $a_{i}$. In many physical considerations, the magnitudes of the eigenvalues of $\hat{A}$ play the crucial role. For example, they determine the energy levels of atoms and, hence, the spectrum of radiation. However, in quantum information the magnitudes are not important. Here $\left(a_{i}\right)$ are just labels. The latter viewpoint is very useful for our applications to DM, see section 5.

By the basic probabilistic law of QM, Born's rule, the probability to get the number $a_{i}$ as the result of a measurement is equal to

$$
p\left(A=a_{i} \mid \psi\right)=\left\langle P_{i} \psi \mid \psi\right\rangle=\left\|P_{i} \psi\right\|^{2} .
$$

If after a measurement of the $A$-observable one plans to perform a measurement of another observable $B$, represented by the Hermitian operator $\hat{B}=\sum_{i} b_{i} P_{i}^{\prime}$, then one needs to know even the output state resulting from the first measurement (through the feedback reaction of the measurement to

\footnotetext{
${ }^{6}$ However, it is not a total Hilbert space.
} 
the initial state): $\psi_{i}=\frac{P_{i} \psi}{\left\|P_{i} \psi\right\|}$. This is nothing else than the quantum version of the classical rule for a probability update. In QM it is known as the projection postulate and measurements inducing feedbacks of such a type are called von Neumann-Lüders measurements.

For the $B$-measurement following the $A$-measurement, the state $\psi_{i}$ plays the same role as the state $\psi$ has played for the $A$-measurement.

Observables of the von Neumann-Lüders class serve well into the general quantum DM scheme which will be presented in section 5. It matches well with statistical (ensemble) interpretations of QM. In principle, it can be used even for the subjective interpretation. However, QBism (for its justification) uses generalized observables given by positive operator valued measures (POVMs) (see Khrennikov (2010); Khrennikov et al. (2014a) for a discussion). This is not just a mathematical peculiarity. This is the crucial point of QBism's treatment of the Born rule (see, for example, Fuchs (2011), Fuchs $\&$ Schack (2013)). Even for an observable $A$ of the von Neumann-Lüders class, to interpret the equality (12), QBists have to proceed with a POVM determining a prior quantum state $\rho$.

\section{Quantum decision making through update of the belief state}

There is given a complex Hilbert space $H$ representing belief states of Alice. There are two given observables $B=\theta_{1}, \ldots, \theta_{m}$ and $A=x_{1}, \ldots, x_{m}$. The first one corresponds to the determination of the state of nature (of mind) and the second one to the collection of additional information (which will be used for PU). Denote the corresponding Hermitian operators as $\widehat{A}$ and $\widehat{B}$. Here, $\widehat{A}=\sum_{x} x E_{x}^{a}$ and $\widehat{B}=\sum_{\theta} \theta E_{\theta}^{b}$, where $\left(E_{\theta}^{b}\right)$ and $\left(E_{x}^{a}\right)$ are orthogonal projectors corresponding to eigen-subspaces of these operators.

We consider the following PU-scheme. Alice creates an initial mental representation of the situation given by a pure quantum state $\psi_{0} \in H$, a belief state (thus here $\left\|\psi_{0}\right\|=1$ ). With the aid of this state, she assigns the subjective probability to the 'states of nature' by performing direct measurements of $B$ and she gets the prior probabilities:

$$
\pi(\theta)=\left\langle E_{\theta}^{b} \psi_{0} \mid \psi_{0}\right\rangle=\left\|E_{\theta}^{b} \psi_{0}\right\|^{2} .
$$

This observation is a process of DM about possible probabilities for $\theta$.

Remark. By introducing the prior probabilities we have to impose the assumption that by performing 'prior-measurement' Alice does not modify 
the initial belief state $\psi_{0}$. It can be perfectly reproduced again to be used for further mental measurements. In comparison to physics, this assumption is natural. In physics it is always assumed that there is a preparation procedure generating an ensemble of systems in the same state. Of course, everybody understands that this is only a theoretical idealization and the real experimental situation is more complicated. Thus, the mental analog of this physical assumption on a state preparation procedure is that the brain which is going to solve some concrete problem, is able to prepare the same belief state (at least approximately (see Khrennikov et al. (2014a)) for a discussion on the stability in the mental state preparation). However, for some mental contexts this assumption may be very restrictive. In principle, it is possible to proceed without it. The determination of the prior probabilities is just a tool of the traditional Bayesian approach. It is really important to start with an assignment of the prior probabilities, since they are explicitly present in the update rule (9). The quantum scheme is about the updates of states and not only probabilities. The state update leads to the posterior probabilities. But the same quantum(-like) state encodes probability distributions for outcomes of incompatible observables. Such distributions cannot be unified without appealing to states since there is no joint probability distribution.

In principle we can proceed without the explicit assignment of the prior probabilities $\pi(\theta)$ given by (13). Thus, the prior measurement of the $B$ observable can be eliminated from the quantum scheme of PU. So, we can start simply with the preparation of the initial belief state $\psi_{0}$ and its update resulting from gaining information with the aid of the $A$-observable, see below.

Now Alice wants to update the probabilities of $\theta$ on the basis of additional information from the measurement of $A$. By using the quantum rule for conditional probabilities we get

$$
\pi(\theta \mid x)=\frac{\left\langle E_{\theta}^{b} E_{x}^{a} \psi_{0} \mid E_{x}^{a} \psi_{0}\right\rangle}{\left\|E_{x}^{a} \psi_{0}\right\|^{2}} .
$$

This is the basic quantum PU rule corresponding to observables of the von Neumann-Lüders type.

In the formalism of quantum PU and DM, instead of a collection $\Theta$ of states of nature (mind), we can consider a collection of hypotheses $\left(H_{k}\right)$ which are represented by projectors forming a mutually orthogonal partition of the unit operator, $\sum_{k} H_{k}=I$. 
As for the classical PU, the probabilities in (14) can be interpreted either as subjective or as frequency based, which leads to two basic interpretations of the quantum state; i) the subjective one structured as QBism (section 7) or ; ii) the statistical one represented by a variety of interpretations in the 'spirit of Copenhagen' or in the spirit of the Einstein ensemble interpretation. See section 6 below for the so-called Växjö interpretation.

By the subjective interpretation, $\psi_{0}$ represents the belief state of Alice (i.e., representing her private beliefs). She first updates this state by measuring the $A$-variable (in general, this is a self-measurement performed unconsciously) and on the basis of this update, she assign new degrees of belief to the values of the parameter $\theta$. We can speculate ${ }^{7}$ that Alice's brain really uses the quantum-like representation of probabilities and that she makes decisions by using odds given by the quantum analog of the classical Bayesian odds (again in the case of the dichotomous $\theta$ ) :

$$
O\left(\theta_{1}\right)=\frac{\pi\left(\theta_{1} \mid x\right)}{\pi\left(\theta_{2} \mid x\right)}=\frac{\left\langle E_{\theta_{1}}^{b} E_{x}^{a} \psi_{0} \mid E_{x}^{a} \psi_{0}\right\rangle}{\left\langle E_{\theta_{2}}^{b} E_{x}^{a} \psi_{0} \mid E_{x}^{a} \psi_{0}\right\rangle}
$$

Thus, we can speculate that Alice's brain really computes (unconsciously) the quantity $O\left(\theta_{1}\right)$ and if it is larger than 1 , she makes the decision that $\theta=\theta_{1}$.

By the statistical interpretation, (14) is not about the internal structure of the process of DM, but it describes the statistical distribution in a long series of DM-experiments.

The use of the subjective interpretation, in particular, of QBism, is more attractive from the viewpoint of modeling cognition. The statistical interpretation can be used to model statistical data obtained in experimental studies in psychology and psychophysics.

Proposition. Quantum PU coincides with classical Bayesian PU iff operators representing observables commute.

Proof. a). Suppose that $[\widehat{A}, \widehat{B}]=0$. In general (regardless of commutativity) by using the quantum rule for conditional probabilities we get

$$
p(x \mid \theta)=\frac{\left\langle E_{x}^{a} E_{\theta}^{b} \psi_{0} \mid E_{\theta}^{b} \psi_{0}\right\rangle}{\left\|E_{\theta}^{b} \psi_{0}\right\|^{2}} ;
$$

\footnotetext{
${ }^{7}$ But just speculate, because quantum-like modeling cannot provide us with deeper insights on the brain's functioning. The brain is treated as a black box.
} 
we also have

$$
p(x)=\left\langle E_{x}^{a} \psi_{0} \mid \psi_{0}\right\rangle=\left\|E_{x}^{a} \psi_{0}\right\|^{2} .
$$

For the quantum PU commutativity of projectors implies:

$\pi(\theta \mid x) p(x)=\left\langle E_{\theta}^{b} E_{x}^{a} \psi_{0} \mid E_{x}^{a} \psi_{0}\right\rangle=\left\langle E_{x}^{a} E_{\theta}^{b} E_{x}^{a} \psi_{0} \mid \psi_{0}\right\rangle=\left\langle E_{\theta}^{b}\left(E_{x}^{a}\right)^{2} \psi_{0} \mid \psi_{0}\right\rangle=\left\langle E_{\theta}^{b} E_{x}^{a} \psi_{0} \mid \psi_{0}\right\rangle$.

In the same way

$p(x \mid \theta) p(\theta)=\left\langle E_{x}^{a} E_{\theta}^{b} \psi_{0} \mid E_{\theta}^{b} \psi_{0}\right\rangle=\left\langle E_{\theta}^{b} E_{x}^{a} E_{\theta}^{b} \psi_{0} \mid \psi_{0}\right\rangle=\left\langle E_{x}^{a}\left(E_{\theta}^{b}\right)^{2} \psi_{0} \mid \psi_{0}\right\rangle=\left\langle E_{x}^{a} E_{\theta}^{b} \psi_{0} \mid \psi_{0}\right\rangle$.

By using commutativity once again we obtain that $\pi(\theta \mid x) p(x)=p(x \mid \theta) p(\theta)$.

b). Suppose that, for any state $\psi_{0}, \mathrm{PU}$ is given by the Bayes rule. This means that

$$
\left\langle\left[E_{x}^{a}, E_{\theta}^{b}\right] \psi_{0} \mid \psi_{0}\right\rangle=0
$$

for any pure state $\psi_{0}$. For a complex Hilbert space this necessarily implies that $\left[E_{x}^{a}, E_{\theta}^{b}\right]=0$.

From the quantum PU rule (14), it is clear that this PU does not idle for $\pi(\theta)=0,1$. Thus, a quantum agent can ignore the Cromwell rule. This is a very important feature of PU in the QP-framework. Quantum PU can lead to novel creative decisions (as opposed to the classical PU which idles for states $\theta$ with zero prior probability).

We remark that in the previous considerations, the initial belief state is a pure state. This assumption is quite natural from the psychological viewpoint: i.e. to start with a superposition of possibilities represented by a pure state. However, the scheme works as well for any initial belief state represented as a most general quantum state, a so-called mixed state, given by a density operator $\rho$. Observables $A$ and $B$ also can be of the most general form given by POVMs. Such generalization is especially important for QBism, section 7, where the $A$-observable has to be an informationally complete POVM.

A comparison of the classical Bayesian and quantum rules for $\mathrm{PU}$ is a novel and interesting topic for experimental research (see Khrennikova (2014); Basieva et al. (2016)) for the first steps in this direction.

\section{Växjö interpretation: inter-relation of Born's rule and FTP}

This interpretation (see Khrennikov (2002)), was born out of an attempt to combine consistently the views of Einstein and Bohr (see, for instance, 
Plotnitsky (2006); Plotnitsky (2009); Khrennikov (2009): realism and contextuality). It is the (ensemble) statistical interpretation. It was born from the observation that, in fact, Bohr's contextuality does not imply nonrealism ${ }^{8}$. Thus, a theory can (but need not) be both contextual and realist. Contextuality has to be treated statistically as contextuality of probabilities, i.e. their dependence on experimental contexts. By the Växjö interpretation, QM is a special mathematical formalism to work with contextual probabilities for families of, in general, incompatible contexts. In particular, by this interpretation the violation of Bell's inequality is simply a consequence of the contextuality of the experimental test (as in the psychological test presented by Conte et al. (2008)). The main distinguishing feature of QP is its complex Hilbert space representation. All quantum contexts can be unified with the aid of a quantum state $\psi$. Thus, QP is not simply a probability model based on a family of Kolmogorov probability spaces labeled by contexts. It contains a 'transcendental element' $\psi$ whose interpretation is still one of the main problems of modern quantum physics. We remark that cognitive processes, in particular, DM are fundamentally contextual. Therefore, contextual probability models (and not only QP) are very useful in applications which model cognition and DM (see, for example, Khrennikov (2010), Dzhafarov \& Kujala (2013, 2014a, b)).

The two-slit experiment (see, e.g. Khrennikov (2010) for a non-physicist

\footnotetext{
${ }^{8}$ In discussions on quantum foundations, the issues of reality and realism are very delicate. 'Copenhageners', Bohr, Heisenberg, Pauli, Dirac, von Neumann, Fock, and others did not deny the reality of, e.g. atoms and electrons. They deny a possibility of a realist description of quantum phenomena: a possibility to treat properties of quantum systems independently of measurement devices. For them QM is complete, i.e. any deeper description of micro-phenomena than given by QM is impossible. This statement is known as leading to the impossibility of introducing hidden variables. In applications of the QM-methods to cognition, the claims about the impossibility of a deeper description than the operational quantum-like one, have to be taken with caution. We cannot ignore the presence of neurophysiological models of the brain functioning (i.e., the modeling by tools from system biology). At this point in time, we are not (yet?) able to connect them with the quantum-like model. However, it would be dangerous to reject a possibility of establishing such connection completely. Therefore, the Växjö interpretation is really useful for cognitive and psychological applications. In contrast to interpretations in the spirit of Copenhagen (see Plotnitsky (2006); Plotnitsky (2009)), this interpretation is not anti-realist (but the price for realism is contextuality). The same can be said about quantum-like modeling in economics. One cannot simply deny the classical models of economics. Here 'hidden parameters' exist as well.
} 
friendly presentation) plays a fundamental role in QP, and it can be considered as a PU procedure. There are given prior probabilities that an electron (photon) $S$ passes each of the slits, $p(i), i=1,2$. Typically, it is assumed that $p(i)=1 / 2$ (the slit configuration which is symmetric with respect to the source). There are also given transition probabilities $q(x \mid i)$ that $S$ is detected at the point $x$ of the registration screen under the assumption that $S$ passed the $i$ th slit. The latter probabilities can be found in experimental contexts $C_{i}, i=1,2$, when only the $i$ th slit is open (we still follow Feynman $\&$ Hibbs (1965) in straightforward fashion). Then, we want to predict the probability $q(x)$ that $S$ is detected at the point $x$ in context $C_{12}$ (when both slits are open). It is convenient to work with discrete observables, so let $x=j=1,2, \ldots$ (i.e. the registration screen is divided into discrete cells).

If the contexts $C_{1}, C_{2}, C_{12}$ are probabilistically compatible, then classical probability theory is applicable through FTP: $q(j)=\sum_{i} p(i) q(j \mid i)$. However, probabilities calculated with the aid of the quantum formalism do not match with the classical FTP. And it is clear why: contexts $C_{i}, i=1,2$, and $C_{12}$ are incompatible. Their incompatibility is a consequence of complementarity, the wave-particle duality. If we represent the probabilities in FTP by using the Born rule, we can easily see that this formula is violated. The statistical data from experiments with electrons and photons also violates FTP. Thus, the classical FTP cannot be used in the quantum framework. Hence, it has to be modified. What would be the most natural modification? It is an additive perturbation of FTP of the form: $q(j)=\sum_{i} p(i) q(j \mid i)+\delta(j)$, where $\delta(j)$ is a perturbation term - 'interference term'. If $\delta(j)=0$, we obtain the classical FTP. We remark that for real data it is more natural to speak, not about the explicit equality to zero, but about the existence of a small $\epsilon>0$ such that $\delta(j)<\epsilon$. In this way we smoothly transit from the classical case (compatible contexts) to the nonclassical case (incompatible contexts). The magnitude of $\delta(j)$ can be interpreted as a measure of deviation from classicality. In QM, $\delta(j)$ has the meaning of the interference term. It can be positive: constructive interference or it can be negative: destructive interference.

The two-slit experiment is just a special example of the quantum probability update via FTP with the interference term involving two incompatible observables, $A$ and $B$. In the two-slit experiment, $A$ is the "what slit passing observable' (expressing the position of $S$ ) and $B$ gives the point of detection on the photo-emulsion screen (expressing the momentum of $S$ ).

Let us now proceed in the abstract framework . To simplify considerations, we restrict (for a moment) considerations to a dichotomous observable 
$A$. We also assume that both $A$ and $B$ are observables of the von NeumannLüders type. Then, it is possible to show that quantum FTP has the following form (see Khrennikov (2010)):

$$
q(j)=\sum_{i} p(i) q(j \mid i)+2 \cos \theta_{j} \sqrt{\prod_{i} p(i) q(j \mid i)},
$$

where $\theta_{j}$ indicates some angles combined of quantum phases. Thus, for observables of the von Neumann-Lüders type, the relative magnitude of the interference term is characterized by the inequality: $\lambda(j)=\frac{\delta(j)}{2 \sqrt{\prod_{i} p(i) q(j \mid i)}} \leq 1$. We can say that for the von Neumann-Lüders observables, the deviation from the classical FTP is relatively small. However, by considering generalized quantum observables given by POVMs, we can obtain $\lambda(j)>1$ (see Khrennikov \& Basieva (2014)). Thus, in the case of a dichotomous observable $A$, the quantum formalism covers violations of FTP for all possible magnitudes. In this case, QM just describes all probability update schemes violating FTP up to some degree (including the classical case where there is no violation). This is a very simple and heuristically clear treatment of $\mathrm{QP}$ as a generalization of the classical statistical inference. In the case of non-dichotomous $A$, the mathematics is more complicated. However, the heuristics are the same.

\section{Subjective interpretation and decision making}

The subjective interpretation of the quantum state and QP is the cornerstone of QBism (see Fuchs \& Schack (2013) (cf. Haven (2008)). Fuchs \& Schack (2015) (p. 3) mentioned that:

"The fundamental primitive of QBism is the concept of experience. According to QBism, quantum mechanics is a theory that any agent can use to evaluate his expectations for the content of his personal experience.

QBism adopts the personalist Bayesian probability theory pioneered by Ramsey (1931) and de Finetti (1990) and is put in modern form by Savage (1954) and Bernardo \& Smith (1994) among others. This means that QBism interprets all probabilities, in particular those that occur in quantum mechanics, as an agent's personal, subjective degrees of belief. This includes the case of certainty - even probabilities 0 or 1 are degrees of belief. .[..].."

Fuchs \& Schack (2015) (p. 4) also mention that: 
"In QBism, a measurement is an action an agent takes to elicit an experience. The measurement outcome is the experience so elicited. The measurement outcome is thus personal to the agent who takes the measurement action. In this sense, quantum mechanics, like probability theory, is a single user theory. A measurement does not reveal a pre-existing value. Rather, the measurement outcome is created in the measurement action."

The subjective interpretation of probability corresponding to the quantum(like) representation of the mental state matches well the quantum(-like) modeling of DM. A human being, say Alice, assigns subjective probabilities to different alternatives in DM. These probabilities, as was emphasized by de Finetti, "do not exist", i.e., they do not have any objective value. They are totally private and represent the degrees of Alice's belief. By getting new information, Alice updates the probabilities by using the 'quantum Bayes rule'. Here probabilities are assigned to individual decisions, e.g. to buy or not to buy this concrete financial asset. This is a very consistent picture and its applicability, not only to cognition modeling, psychology, psychophysics, economics, but even to molecular biology was discussed in very much detail by Asano et al. (2015).

We repeat that the use here of the nonclassical rule for PU is crucial. One cannot approach this rule just by following a classical subjectivist line of reasoning, e.g. by following de Finetti. We really have to combine, as was proposed by Fuchs, de Finetti's subjective probability with Born's rule and by exploring some mathematics of symmetric informationally complete positive operator valued measures, to derive a new version of FTP, QBism's FTP (see Fuchs \& Schack (2015)). Thus, Alice following Fuchs, makes PU and hence her DM is different from Alice's following de Finetti. Another important contribution of the quantum formalism is that it describes not only probabilities but also states. In the modeling of cognition and DM it is very natural to associate these quantum-like states with belief-states (mental-states). The private agent's perspective advertised by QBism is again important for us. Classical subjective probability does not say anything about the belief-states of agents. They are expressed very roughly as subjective probabilities. The main distinguishing feature of the quantum representation of states (both in physics and cognition and DM modeling) is that they encode not only probabilities for possible results of compatible observables, but even of incompatible observables.

We can conclude that, although QBism is not so widely accepted in the quantum physical community and, in particular, it was criticized by Khren- 
nikov (2002), it seems to be a very natural candidate to interpret QP in applications to cognition and DM.

As was pointed out, in spite of its tremendous success, nowadays QBism also forms the subject of critiques from many top quantum physicists - those who claim that they 'do real physics' and do not meddle with private beliefs. Some of them even treat QBism as a form of solipsism. We shall present some arguments defending the use of the subjective probability interpretation in physics. The coming discussion is not so important for applications of QBism to cognition and DM.

The main question which is often asked about QBism by quantum physicists is: Where does the subjective probability come from? As was remarked, this question can be in principle ignored in applications of QBism to cognition. We also remark that this question is generally about subjective probability (without any classical/quantum distinction). In principle, it might be discussed in section 3. But it became really actual and unescapable in the quantum framework since subjective probability is not frequently used in classical physics.

In quantum physics C. Fuchs tries to justify the private agent perspective with the aid of the objective indeterminism of nature (see James (1882, 1884)). Thus, although subjective probabilities are personal, they have some degree of objectivity, as representing nature's objective indeterminism. The latter has some similarity with von Neumann's concept of irreducible quantum randomness. However, the concept of objective indeterminism is more general. In particular, in contrast to irreducible quantum randomness, it has no explicit relation to QM. James invented this concept in 1882 (or maybe even earlier), long before physicists started to deal with the peculiarity (in the form of acausality) of the randomness of outputs of quantum measurements. For James, indeterminism is a general feature of nature, whether physical or mental. In some sense Fuchs just wants to restrict the domain of action of Jamesian indeterminism to the quantum world and in this way he finds the objective justification of QBism. In some sense the domain of action of objective indeterminism and hence Bayesianism is not restricted to quantum physics: classical statistical physics also has to be treated from the personal agent's perspective.

We conclude that, in spite of the use of the subjective interpretation of the quantum state and probability, QBism is not a form of solipsism. QBism's indeterminism is objective: the objectivity of nature is exhibited in its ability to produce randomness (see again James $(1882,1884)$ ). 
In this paper we are interested in applications of QBism to cognition and DM and, as was mentioned, we can proceed without the objectification of subjective probabilities which Alice assigns to possible decisions.

\section{Statistical interpretation and decision making}

By using the statistical interpretation, in general, we cannot associate $\mathrm{QP}$ with the concrete mental state (belief state) of Alice. In QM we consider an ensemble (and typically it is very large, say a few million photons) of quantum systems prepared in the same state. Born's rule then encodes the prediction about the probabilities, treated statistically, of the results of possible measurements. The application of this general statistical setting to DM and problem solving gives us the following picture. Alice does not make decisions by assigning probabilities in the concrete act of DM. The process of selection of one of the possible alternatives in DM is not so straightforward. The quantum(-like) model does not provide a consistent picture of this process. This is a purely operational model which describes probabilities for decision-alternatives. Probabilities have the meaning of frequencies for an ensemble of decision makers.

Although the above operational scheme is completely sufficient for work-

ing with statistical data, one may dream for a deeper clarification of the DM mechanism. Here we can proceed similarly to von Neumann (1955) and assume that the concrete mental state of Alice encodes a kind of irreducible quantum(-like) randomness. Thus, again by following von Neumann, we can treat statistical probabilities-frequencies as simply the ensemble representation of this intrinsic randomness. However, one has to extend von Neumann's thesis about such a randomness to the mental world. During the 1930's-1990's when the physical and mental worlds were considered (more or less following Descartes' duality principle) as two totally different worlds, such an extension would have been deemed to be unacceptable.

Another possibility is to follow C. Fuchs and explore James' objective indeterminism, but combine it with von Neumann's idea that QP represents its statistical-frequency realization. Thus here, in the von Neumann-Fuchs scheme, a decision has in general a nontrivial contribution of genuine mental randomness, cf. section 7 .

However, by keeping the statistical interpretation we do not have to be coupled to this scheme, i.e. to take into account the genuine mental randomness. In the more pragmatic version of the Copenhagen interpretation 
of QM due to Bohr, Pauli and recently Plotnitsky, one does not try to 'explain' the origin of quantum randomness. It is considered as an empirical fact that quantum probabilities, averages, and correlations are the result of our inability to know some hidden parameters. In applications to cognition, psychology and psychophysics, we can remain on the same position, and say that empirical data demonstrated that such Statistical Copenhagen Interpretation (SCI) provides the most consistent (at least for now) interpretation of the use of QP in DM.

By using the Växjö interpretation one can proceed with hidden variables, but they have to be of the contextual type, i.e. they cannot be assigned to a system independently of the experimental context. Such a model can be local, but contextuality also can lead to violation of Bell's inequality. By applying this interpretation to QP-DM we come to the contextual probability viewpoint of DM. As in SCI, there is no need for objective indeterminism, irreducible quantum randomness, to explain deviations of QP-DM from CPDM.

\section{Concluding remarks}

Both in QBism and the Växjö interpretation, the quantum probabilistic formalism is treated as PU machines. These machines are nonclassical (see (21) for the FTP used in the Växjö interpretation and see (Fuchs \& Schack (2015)) for the corresponding FTP-like rule of QBism).

At present, it is difficult to select one concrete interpretation. However, we hope that our analysis has clarified the problem of the interpretation of probability in QP-DM and that it will stimulate other researchers to contribute to this exciting and novel field of research: the foundations of the quantum-like approach to DM.

\section{Acknowledgments}

AK was supported by the grant 'Mathematical Modeling of Complex Hierarchic Systems' of the Faculty of Technology, Linnaeus University. AK and EH want to thank in particular Ehti Dzhafarov for organizing the 2014 Winer Memorial Lecture (at Purdue University). At this wonderful event many discussions were held on the topic of how probability can be interpreted in QP-DM. 


\section{References}

Aerts, D., Sozzo, S., \& Tapia, J. (2012). A quantum model for the Ellsberg and Machina paradoxes. In J. Busemeyer et al. (Eds.), Quantum interaction 2012. Lecture Notes in Computer Science (pp. 48-59). Berlin: Springer.

Aerts, D., Sozzo, S., \& Veloz, T. (2015). The quantum nature of identity in human concepts: Bose-Einstein statistics for conceptual indistinguishability. International Journal of Theoretical Physics, 54 (12), 4430-4443.

Asano, M., Khrennikov, A., Ohya, M., Tanaka, Y., \& Yamato, I. (2015). Quantum adaptivity in biology: from genetics to cognition. Heidelberg-Berlin-New York: Springer.

Atmanspacher, H., Haven, E., Kitto, K., \& Raine, D. (2014). Quantum Interaction: 7th International Conference, Quantum Interaction 2013. Lecture Notes in Computer Science 8369.

Atmanspacher, H., \& Filk, T. (2014). The relevance of Bell-type inequalities for mental systems. In H. Atmanspacher et al. (Eds.), Quantum Interaction 2013. Lecture Notes in Computer Science (pp. 231-243). Berlin: Springer.

Atmanspacher, H., \& Filk. T. (2014). Non-commutative operations in consciousness studies. Journal of Consciousness Studies, 21 (3/4), 24-39.

Bagarello, F. (2012). Quantum dynamics for classical systems: with applications of the number operator. New York: J. Wiley.

de Barros, A. J., \& Suppes, P. (2009). Quantum mechanics, interference and the brain. Journal of Mathematical Psychology, 53, 306-313.

de Barros, A. J., \& Oas, G. (2014). Negative probabilities and counter-factual reasoning in quantum cognition. Physica Scripta, T163, 014008.

de Barros, A. J., \& Oas, G. (2015). Some examples of contextuality in physics: implications to quantum cognition. In E. Dzhafarov, R. Zhang, \& S. M. Jordan (Eds.), Contextuality from quantum physics to psychology. Singapore: World Scientific Publishers.

Basieva, I., Pothos, E., Trueblood, J., Khrennikov, A., \& Busemeyer, J. (2016). Quantum probability updating from zero prior (by-passing Cromwell's rule), submitted to Journal of Mathematical Psychology. 
Bell, J. S. (1987). Speakable and unspeakable in quantum mechanics. Cambridge: Cambridge University Press.

Boole, G. (1958). An investigation of the laws of thought. New York: Dover Edition.

Bernardo, J. M., \& Smith, A. F. M. (1994). Bayesian theory. Chichester: J. Wiley.

Busemeyer, J. R., \& Bruza, P. D. (2012). Quantum models of cognition and decision. Cambridge: Cambridge University Press.

Busemeyer, J.R., Wang, J., Khrennikov, A., \& Basieva, I. (2014). Applying quantum principles to psychology. Physica Scripta, T163, 014007.

Conte, E., Khrennikov, A., Todarello, O., Federici, A., Mendolicchio, L., \& Zbilut, J. P. (2008). A preliminary experimental verification on the possibility of the Bell inequality violation in mental states. Neuroquantology, 6, 214-221.

de Finetti, B. (1990). Theory of probability. New York: J. Wiley.

Dzhafarov, E.N., \& Kujala, J.V. (2013). All-possible-couplings approach to measuring probabilistic context. PLoS One, 8(5): e61712.

Dzhafarov, E.N., \& Kujala, J.V. (2014). On selective influences, marginal selectivity and Bell/CHSH inequalities. Topics in Cognitive Science , 6, 121-128.

Dzhafarov, E.N., \& Kujala, J.V. (2014). No-Forcing and No-Matching theorems for classical probability applied to quantum mechanics. Foundations of Physics, 44, 248-265.

Ezhov, A. A., \& Berman, G. P. (2003). Introduction to quantum neural technologies. Paramus: Rinton Press.

Feller, W. (1968). An introduction to probability theory and its applications. New York: Vol. 1. J. Wiley \& Sons.

Feynman, R., \& Hibbs, A. (1965). Quantum mechanics and path integrals. New York: McGraw-Hill.

Fuchs, C.A. (2011). Interview with a quantum Bayesian. In M. Schlosshauer (Ed.), Elegance and enigma: the quantum interviews. Springer Frontiers Collection (pp. 1-20). Berlin: Springer. 
Fuchs, C.A., \& Schack, R. (2013). Quantum-Bayesian coherence. Review of Modern Physics, 85, 1693-1715.

Fuchs, C. A., \& Schack, R. (2015). QBism and the Greeks: why a quantum state does not represent an element of physical reality. https://pure.royalholloway.ac.uk/portal/files/23282396/FuchsSchack.pdf

Gisin, N., \& Aspect, A. (2014). Quantum chance: nonlocality, teleportation and other quantum marvels. Berlin: Springer.

Haven, E. (2008). Private information and the 'information function': a survey of possible uses. Theory and Decision, 64, 193-228.

Haven, E., \& Khrennikov, A. (2013). Quantum social science. Cambridge: Cambridge University Press.

Hensen, B. et al. (2015). Loophole-free Bell inequality violation using electron spins separated by 1.3 kilometres. Nature, 526, 682-686

James, W. (1882). On some Hegelisms. In: The will to believe and other essays in popular philosophy. The Project Gutenberg Ebook (2009)(pp. 263-298). www.gutenberg.net.

James, W. (1884). The dilemma of determinism. In: The will to believe and other essays in popular philosophy. The Project Gutenberg Ebook (2009)(pp. 145183). www.gutenberg.net.

Khrennikov, A. (2002). The Växjö Interpretation of Quantum Mechanics. In: Quantum theory: reconsideration of foundations. Växjö: Växjö University Press (pp.163-170). arxiv.org/abs/quant-ph/0202107.

Khrennikov, A. (2008). Bell-Boole inequality: nonlocality or probabilistic incompatibility of random variables? Entropy, 10, N 2, 19-32.

Khrennikov, A. (2009). Interpretations of probability. Berlin: De Gruyter (second edition (completed)).

Khrennikov, A. (2010). Ubiquitous quantum structure: from psychology to finance. Berlin-Heidelberg-New York: Springer. 
Khrennikov, A., Basieva, I., Dzhafarov, E.N., \& Busemeyer, J.R. (2014). Quantum models for psychological measurements: an unsolved problem. PLoS ONE, 9, Article ID: e110909.

Khrennikov, A., Ramelow, S., Ursin, R., Wittmann, B., Kofler, J., \& Basieva, I. (2014). On the equivalence of the Clauser-Horne and Eberhard inequality based tests. Physica Scripta, T163, 014019.

Khrennikov, A., \& Basieva, I. (2014). Quantum model for psychological measurements: from the projection postulate to interference of mental observables represented as positive operator valued measures. NeuroQuantology, 12, 324-336.

Khrennikova, P. (2014). A quantum framework for 'sour grapes' in cognitive dissonance. In: H. Atmanspacher et al. (Eds.), Quantum Interaction 2013. Lecture Notes in Computer Science (pp. 270-280). Berlin: Springer.

Kolmogorov, A. N. (1933). Grundbegriffe der Wahrscheinlichkeitsrechnung. Berlin: Springer-Verlag (English translation: Kolmogorov, A.N. (1956). Foundations of the theory of probability. New York: Chelsea Publishing Company)

Lindley D. (1991). Making decisions (2nd Ed.). Wiley Publishing.

Mermin, N. D. (2014). Why QBism is not the Copenhagen interpretation and what John Bell might have thought of it. arXiv:1409.2454v1 [quant-ph].

Plotnitsky, A. (2006). Reading Bohr: physics and philosophy. Dordrecht: Springer.

Plotnitsky, A. (2009). Epistemology and probability: Bohr, Heisenberg, Schrödinger and the nature of quantum-theoretical thinking. Heidelberg-BerlinNew York: Springer.

Plotnitsky, A. (2014). Are quantum-mechanical-like models possible, or necessary, outside quantum physics? Physica Scripta, T163, 014011.

Ramsey, F. P. (1931). In R. B. Braithwaite (Ed), The foundations of mathematics and other logical essays (pp. 156-198). New York: Harcourt, Brace and Company.

Rocchi, P. (2003). The structural theory of probability. New ideas from computer science on the ancient problem of probability interpretation. New York: Springer. 
Rocchi, P. (2014). Janus-faced probability. Heidelberg-Berlin-New York: Springer.

Savage, L. J. (1954) The foundations of statistics. New York: J. Wiley.

Sozzo, S. (2015). Conjunction and negation of natural concepts: a quantumtheoretic framework. Journal of Mathematical Psychology, 66, 83-102.

von Mises, R. (1957). Probability, statistics and truth. London: Macmillan.

von Neumann, J. (1955). Mathematical foundations of quantum mechanics. Princeton: Princeton University Press.

Zeilinger, A. (2010). Dance of the photons: from Einstein to quantum teleportation. New York: Straus and Giroux. 